\author{
by \\ Christopher L. Rumsey \\ NASA Langley Research Center \\ Mail Stop 128, Hampton, VA 23681-0001
}

Paper presented at the

2nd AIAA/CEAS Aeroacoustics Conference May 6-8, 1996

State College, PA 
. 


\title{
COMPUTATION OF ACOUSTIC WAVES THROUGH SLIDING-ZONE INTERFACES USING AN EULER/NAVIER-STOKES CODE
}

\author{
Christopher L. Rumsey* \\ NASA Langley Research Center, Mail Stop 128, Hampton, VA 23681-0001
}

\begin{abstract}
The effect of a patched sliding-zone interface on the transmission of acoustic waves is examined for two- and three-dimensional model problems. A simple but general interpolation scheme at the patched boundary passes acoustic waves without distortion, provided that a sufficiently small time step is taken. A guideline is provided for the maximum permissible time step or zone speed that gives an acceptable error introduced by the sliding-zone interface.
\end{abstract}

\section{Introduction}

The prediction and control of ducted fan noise are important elements of the NASA Advanced Subsonic Technology Noise Reduction Program. Current methods of prediction rely extensively on field measurements and analytical scaling techniques. As computers continue to become more powerful, Euler and NavierStokes computer codes for ducted-fan noise prediction have become increasingly affordable. Recent advances in algorithms ${ }^{1,2,3}$ that can enhance the efficiency of time-accurate Euler and Navier-Stokes computations also make difficult computations, such as three-dimensional (3D) rotor-stator interactions, better suited for inclusion in future engine design cycles.

Previous work by Rai ${ }^{4}$ and Gundy-Burlet et al. ${ }^{5} \mathrm{dem}$ onstrated the feasibility of using Navier-Stokes computations for time-accurate rotor-stator interactions. Gundy-Burlet et al. utilized a combination of overlapping and patched grids; the motion of the rotor relative to the stator was accomplished by "sliding" the rotor grid system past the stator grid system and utilizing a non-conservative linear interpolation to transfer information between the two grid systems. Hall and Delaney ${ }^{6}$ employed a similar patched sliding-zone interface strategy to compute ducted prop-fan flows. Chen and Chakravarthy ${ }^{7}$ utilized patched sliding-zone interfaces to perform rotor-stator computations. They used a simple piecewise-constant projection of flow variables between grid zones, with an area-weighting strategy. Janus and Whitfield ${ }^{8}$ utilized localized grid distortion to pass information between zones that move relative to one another in a prop-fan simulation. Rather than em-

\footnotetext{
*Fluid Mechanics and Acoustics Division, Senior Member AIAA.
}

Copyright (c) 1996 by the American Institute of Aeronautics and Astronautics, Inc. No copyright is asserted in the United States under Title 17, U.S. Code. The U.S. Government has a royalty-free license to exercise all rights under the copyright claimed herein for Govern mental purposes. All other rights are reserved by the copyright owner. ploy interpolation, grid points near the zone interface were distorted, then "clicked" to new positions when appropriate. In refs. 4-8, the focus of the computations was the prediction of global aerodynamic characteristics. Rangwalla and Rai ${ }^{9}$ compared the numerically calculated tonal acoustics with theoretical values for a twodimensional (2D) rotor-stator interaction. Emphasis was placed on the effects of boundary conditions and boundary extent; however, the effect of the patched slidingzone interface on the accuracy of simulating the passage of acoustic waves was not explored.

Because accurate prediction of acoustic waves is essential to any noise-prediction analysis, the effect of the sliding-zone interface on the passage of such waves must be addressed. In the present paper, the effect of a patched sliding-zone interface similar to that employed in refs. 5-7 is examined for several $2 \mathrm{D}$ problems, as well as for the passage of typical rotor-stator interaction modes through a 3D duct with a rotating zone. The effects of time step and speed of the moving zone are examined; an engineering rule of thumb for maximum permissible time step or zone speed is developed.

\section{Governing Equations}

The compressible thin-layer Navier-Stokes equations, written in an inertial reference frame in generalized coordinates, are

$$
\frac{\partial \hat{Q}}{\partial t}+\frac{\partial\left(\hat{F}-\hat{F}_{v}\right)}{\partial \xi}+\frac{\partial\left(\hat{G}-\hat{G}_{v}\right)}{\partial \eta}+\frac{\partial\left(\hat{H}-\hat{H}_{v}\right)}{\partial \zeta}=0
$$

where

$$
\hat{Q}=\frac{Q}{J}
$$

and $Q$ is the vector of conserved variables given as 


$$
Q=\left[\begin{array}{c}
\rho \\
\rho u \\
\rho v \\
\rho w \\
e
\end{array}\right]
$$

The inviscid flux terms are

$$
\begin{gathered}
F=\frac{1}{J}\left[\begin{array}{c}
\rho U \\
\rho U u+\xi_{x} p \\
\rho U v+\xi_{y} p \\
\rho U w+\xi_{z} p \\
(e+p) U-\xi_{,} p
\end{array}\right] \\
\hat{G}=\frac{1}{J}\left[\begin{array}{c}
\rho V \\
\rho V u+\eta_{x} p \\
\rho V v+\eta_{y} p \\
\rho V w+\eta_{z} p \\
(e+p) V-\eta_{t} p
\end{array}\right]
\end{gathered}
$$

and

$$
\hat{H}=\frac{1}{J}\left[\begin{array}{c}
\rho W \\
\rho W u+\zeta_{x} p \\
\rho W v+\zeta_{y} p \\
\rho W w+\zeta_{i} p \\
(e+p) W-\zeta_{x} p
\end{array}\right]
$$

The variable $J$ represents the Jacobian of the transformation

$$
J=\frac{\partial(\xi, \eta, \zeta)}{\partial(x, y, z)}
$$

and the contravariant velocities are given by

$$
\begin{aligned}
& U=\xi_{x} u+\xi_{y} v+\xi_{z} w+\xi_{1} \\
& V=\eta_{x} u+\eta_{y} v+\eta_{z} w+\eta_{t}
\end{aligned}
$$

and

$$
W=\zeta_{x} u+\zeta_{y} v+\zeta_{z} w+\zeta_{1}
$$

The viscous terms are

$$
\hat{F}_{y}=\frac{1}{J}\left[\begin{array}{c}
0 \\
\xi_{x} \tau_{x x}+\xi_{y} \tau_{x y}+\xi_{z} \tau_{x z} \\
\xi_{x} \tau_{y x}+\xi_{y} \tau_{y y}+\xi_{z} \tau_{y z} \\
\xi_{x} \tau_{z x}+\xi_{y} \tau_{z y}+\xi_{z} \tau_{z z} \\
\xi_{x} b_{x}+\xi_{y} b_{y}+\xi_{z} b_{z}
\end{array}\right]
$$

$$
\hat{G}_{v}=\frac{1}{J}\left[\begin{array}{c}
0 \\
\eta_{x} \tau_{x x}+\eta_{y} \tau_{x y}+\eta_{z} \tau_{x=} \\
\eta_{x} \tau_{y x}+\eta_{y} \tau_{y y}+\eta_{z} \tau_{y z} \\
\eta_{x} \tau_{z x}+\eta_{y} \tau_{z y}+\eta_{z} \tau_{z z} \\
\eta_{x} b_{x}+\eta_{y} b_{y}+\eta_{z} b_{z}
\end{array}\right]
$$

and

$$
\hat{H}_{v}=\frac{1}{J}\left[\begin{array}{c}
0 \\
\zeta_{x} \tau_{x x}+\zeta_{y} \tau_{x y}+\zeta_{z} \tau_{x z} \\
\zeta_{x} \tau_{y x}+\zeta_{y} \tau_{y y}+\zeta_{z} \tau_{y z} \\
\zeta_{x} \tau_{z x}+\zeta_{y} \tau_{z y}+\zeta_{z} \tau_{z:} \\
\zeta_{x} b_{x}+\zeta_{y} b_{y}+\zeta_{z} b_{z}
\end{array}\right]
$$

where

$$
b_{x_{i}}=u_{j} \tau_{x_{i} x_{j}}-\dot{q}_{x_{i}}
$$

and $\tau$ and $\dot{q}$ are the shear stress and heat flux terms, respectively. When the thin-layer assumption is invoked, the viscous cross-derivative terms in $\xi, \eta$, and $\zeta$ are neglected.

For the applications here, only the inviscid (Euler) equations are employed. The equations are closed by the equation of state for a perfect gas

$$
p=(\gamma-1)\left[e-\rho \frac{\left(u^{2}+v^{2}+w^{2}\right)}{2}\right]
$$

\section{Description of the Code}

The computer code CFL3D ${ }^{10}$ solves the $3 \mathrm{D}$ time-dependent thin-layer Navier-Stokes equations with an upwind finite-volume formulation. This code can solve flows over multiple-zone grids that are connected in a one-to-one, patched, or overset manner, and can employ grid sequencing, multigrid, and local time stepping in accelerating convergence to steady state. Upwind-biased spatial differencing is used for the inviscid terms, and flux limiting is used to obtain smooth solutions in the vicinity of shock waves. Viscous terms, when employed, are centrally differenced. The equations are solved implicitly with the use of a three-factor approximate factorization (AF). Either the flux-difference-splitting (FDS) method of Roe ${ }^{11}$ or the flux-vector-splitting (FVS) method of Van Leer ${ }^{12}$ can be employed to obtain fluxes at the cell faces. The FDS approach is used for all results in this paper. 


\section{Time-Advancement and Subiteration Algorithm}

The CFL3D code is advanced in time with an implicit AF method. The implicit spatial derivatives are first-order accurate, which results in block-tridiagonal inversions for each sweep. However, for solutions that utilize FDS the block-tridiagonal inversions are further simplified with a diagonal algorithm; when the viscous terms are used, a spectral radius scaling is employed.

Second-order temporal accuracy for a single-step AF scheme is forfeited for unsteady computations with these simplifications to the left-hand side. One method for recovering the desired accuracy is through the use of subiterations. Two subiteration strategies have been implemented in CFL3D. These strategies were explored in detail by Rumsey et al. ${ }^{3}$ The method employed for the computations in this paper is termed the "pseudo time subiteration" (or " $\tau$-TS") method, which uses a secondorder-accurate temporal discretization. In the literature (e.g., Venkateswaran and Merkle ${ }^{13}$ ), the $\tau$-TS method is also referred to as the "dual time" method because a pseudo time is used to iterate to the next desired physical time. The chief advantage to the $\tau$-TS subiteration strategy is that it frees the user from numerical constraints on the time step; an appropriate physical time step can be selected to resolve the physics of the flow in question.

\section{Dynamic Patched-Grid Algorithm}

A patched-grid interface implies that two zones share a common interface at which the grid points do not necessarily connect in a one-to-one manner. For grids in relative motion to one another (for example, when a grid zone around a rotor slides past a grid zone around a stator), dynamic patched interfacing is a relatively easy way to handle the transfer of time-accurate data between zones.

In CFL3D, the patching is accomplished non-conservatively in the following way. First, at the patched interface, the problem is reduced by 1 degree of freedom because data are used only in the two planes of cell centers in computational space nearest each interface to update the ghost-cell boundary conditions in the other zone. For example (as shown in figure 1 in two dimensions), if the $k=k d i m$ boundary of zone 1 interfaces with the $k=1$ boundary of zone 2 , then zone 2 obtains information for its two ghost cells from the $k=k d i m-1$ and $k=k d i m-2$ cell centers of zone 1 ; zone 1 obtains information for its two ghost cells from the $k=1$ and $k=2$ cell centers of zone 2 .

Interpolation is accomplished dynamically at each time step (every time the moving zone changes position). For each face center point on the interface in the "to" zone, a corresponding real-valued index location is determined in the "from" zone. The primitive variables are then interpolated to this real-valued index location. A simple $2 \mathrm{D}$ example is given in figure 2 ; zone 2 acts as the "to" zone, and only one row of ghost cells is shown in the "from" zone for clarity. In this example, the center point of the cell $j=1$ in zone 2 lines up with $\eta=0.6667$ in zone 1 , which lies in the cell $j=1$ of zone 1 . Therefore, this particular ghost-cell boundary condition is interpolated by using the primitive variables at $j=1$ in zone 1 and an appropriately weighted fraction of the $\eta$ direction gradient at $j=1$. Further details of the patchedgrid algorithm are given in Biedron and Thomas. ${ }^{14}$

When periodic boundary conditions are applied and

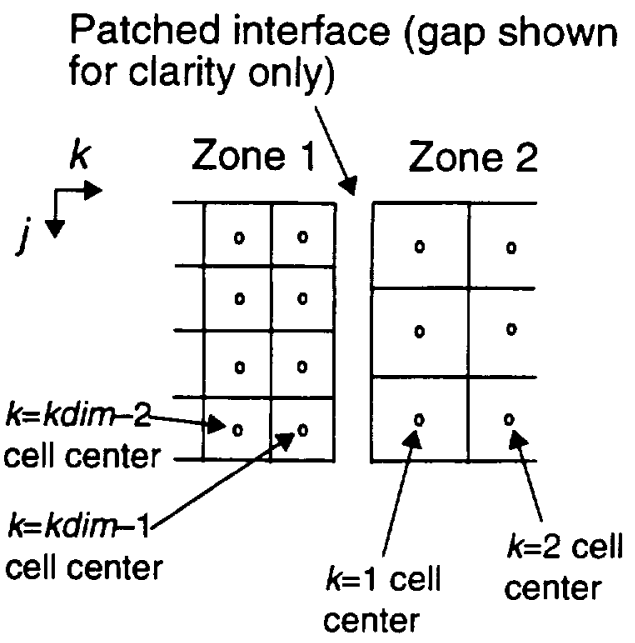

Fig. 1. Sample 2D patched interface.

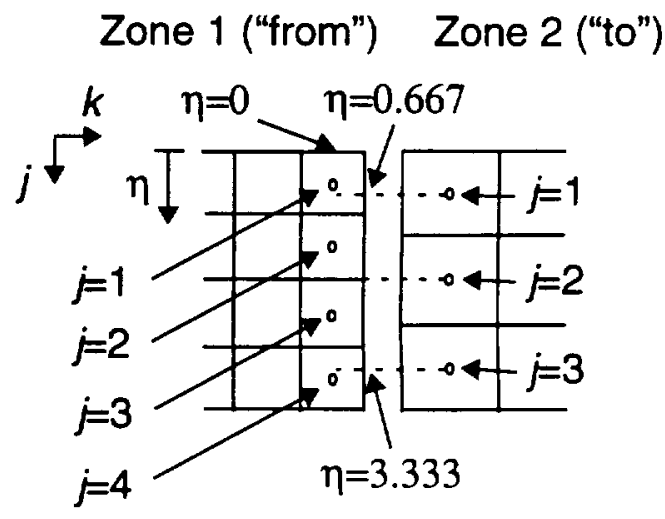

Fig. 2. Sample 2D patched interface, with zone 2 as "to" zone.

one grid zone slides relative to another, copies of both the moving and nonmoving zones must be supplied, which are translated or rotated appropriately to ensure that each "to" zone has a "from" neighbor at all times and vice versa. To avoid the necessity of making too many copies over long times, the current algorithm also periodically rotates or translates the moving block and its solution whenever its movement exceeds a specified limit.

\section{Results}

Several 2D and 3D model problems are examined to study the effect of a patched sliding-zone interface on the passage of acoustic waves. Unless otherwise noted, three $\tau$-TS subiterations with three levels of multigrid are used for the results in this paper. 


\section{D Axially-Moving Acoustic Waves}

The first test case involves acoustic waves that are moving axially in two dimensions $(x, z)$. At the left end of a computational domain, waves are generated by perturbing the free-stream pressure levels in accordance with

$$
p=p_{0}+h \cos \left(\frac{2 \pi t}{T_{1}}\right) \cos \left(\frac{2 \pi z}{T_{z}}\right)
$$

where $p_{0}$ is the nondimensional free-stream static pressure $(=1 / \gamma)$ and $h$ is taken as 0.04518 . Time is nondimensionalized by unit length and speed of sound. The free-stream is quiescent.

This test case is patterned after a computation by Khan, ${ }^{15}$ who used a perturbational form of the Euler equations to compute the nonlinear behavior of a plane acoustic wave that was propagating axially; the results were compared with the theoretical predictions of Pierce ${ }^{16}$ at locations prior to where nonlinear steepening causes shock formation. In the current study, a sinusoidal transverse variation of pressure (in the $z$ direction) is also imposed to test the effect of a sliding-zone interface (no effect is realized otherwise). Comparison with the theoretical results is made at a transverse location at which the acoustic wave front is aligned with the $z$-axis. The variation in the $z$ direction is gradual enough to allow the wave to approximate a plane wave at this location.

The computation is performed by using either one or two zones. In the latter case, zone 2 slides in the $z$ direction along one end of zone 1 ; a patched sliding-zone interface connects the two zones. (See figure 3.) The upper and lower boundaries are treated as periodic interfaces, and the right boundary employs a characteristic boundary condition. To minimize the possibility of spurious reflections from the right boundary, the grid is stretched in the $x$ direction past $x=1.6345$.

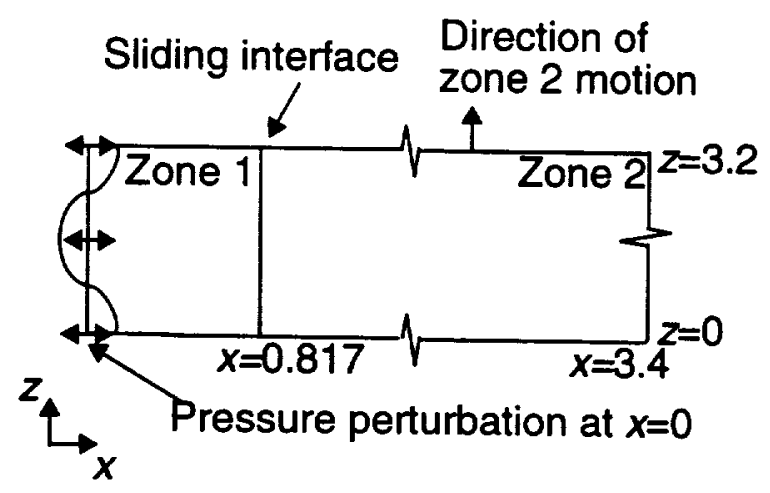

Fig. 3. Sketch of $2 \mathrm{D}$ case for axially-moving acoustic waves.

The parameter $T$, is taken as 0.55164 (which corresponds to a frequency of $0.6 \mathrm{kHz}$ in air), and $T_{z}$ is taken as 3.2. This latter parameter yields one complete period of pressure fluctuation between $z=0$ and $z=3.2$.

Prior to evaluating the effect of the sliding-zone interface, parametric studies were used to determine the grid size and time step necessary to capture the physics of this flow field with the CFL3D code. Figure 4 shows the effect of varying the number of cells in the $z$ direction from 8 to $64 ; 16$ to 32 grid points are sufficient to accurately depict the sinusoidal variation in the acoustic pressure.

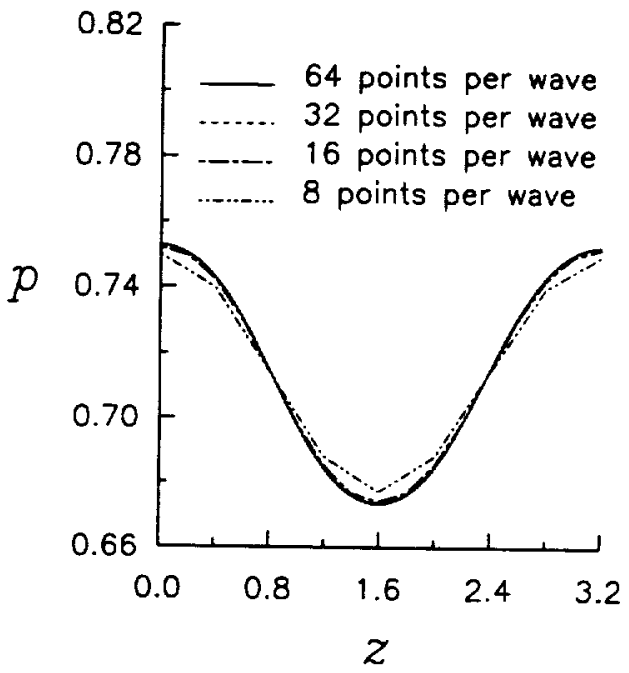

Fig. 4. Effect of number of points in $z$ direction on pressures at $x=0.83$, with $\Delta x=0.01277, \Delta t=0.005$, and $T=3.6$.

The effect of $\Delta x$ is shown in figures 5 and 6 . The wavelength in the $x$ direction for this case is 0.552 . Using a constant $\Delta z$ spacing of 0.1 (32 points per wave in the $z$ direction), the $\Delta x$ spacing is varied from 0.02554 (approximately 22 points per wave) to 0.006385 (approximately 86 points per wave). Figure 5 shows the pressure as a function of $x / \bar{x}$ along $z=0$, where $\bar{x}$ is the value of the distance at which the shock forms:

$$
\bar{x}^{\prime}=\left(\frac{2}{\gamma+1}\right) \frac{\rho^{\prime} a^{\prime 2}}{k^{\prime} h^{\prime}}
$$

(' indicates a dimensional quantity). In this equation, $k^{\prime}=\omega^{\prime} / a^{\prime}, \omega^{\prime}$ is the frequency in $\mathrm{rad} / \mathrm{s}, \rho^{\prime}$ is the density, and $a^{\prime}$ is the speed of sound. For this case, $\bar{x}=1.6194$. Figure 6 shows the amplitude of the fundamental and the first two harmonics as a function of distance. The computational curves in this figure are generated by establishing a periodic solution, computing over a nondimensional time of 1.1 , and using a Fourier decomposition on the time histories of pressure at each spatial location. Three higher harmonics are computed; only the first two are shown in figure 6 for clarity. The predicted fundamental and the first two harmonics agree well with the theoretical predictions of Pierce 16 with 43 or more points per wave. With only 22 points per wave, significant error is realized as $x / \bar{x}$ approaches 1 , particularly for the higher harmonics. 


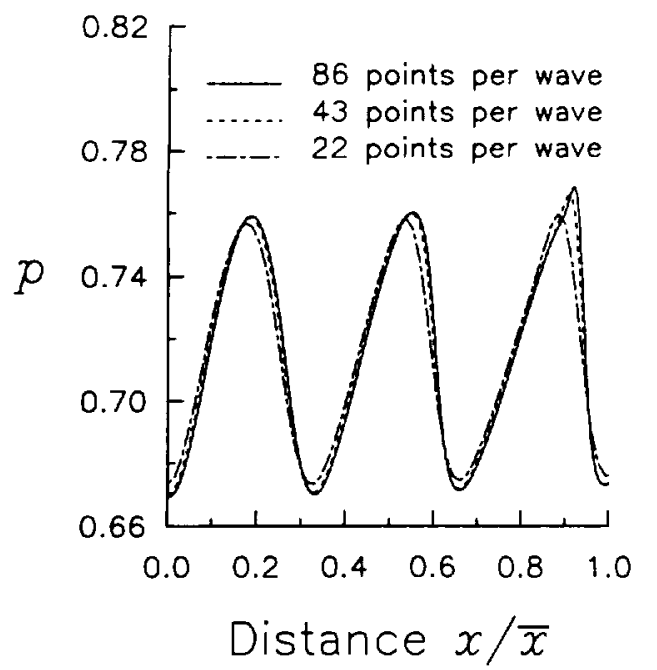

Fig. 5. Effect of number of points in $x$ direction on pressures at $z=0$, with $\Delta z=0.1, \Delta t=0.005$, and $T=3.6$.

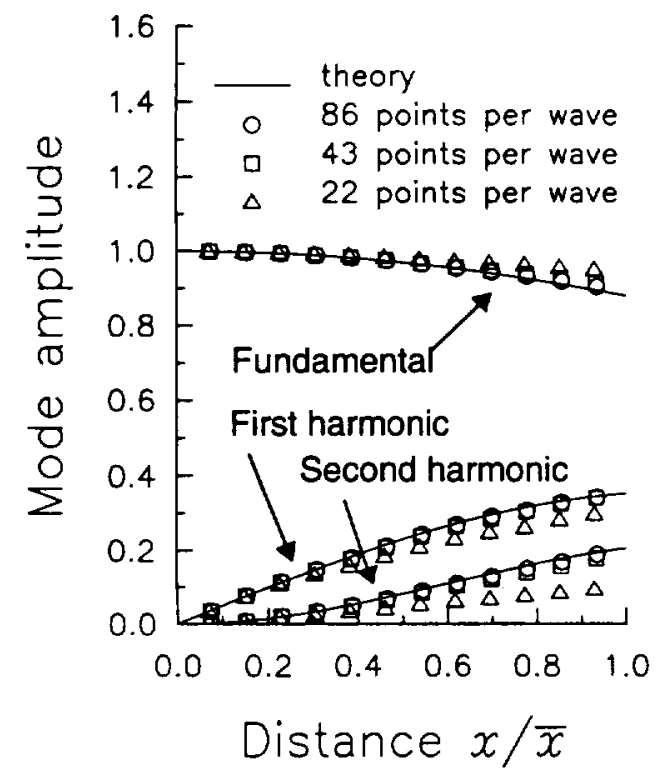

Fig. 6. Effect of number of points in $x$ direction on harmonics at $z=0$, with $\Delta z=0.1$ and $\Delta t=0.005$.

The effect of time step is shown in figure 7. A time step of $\Delta t=0.01$ ( 55 steps per cycle) is sufficient to capture the fundamental and first harmonic but is not sufficient for the higher harmonics. A time step of $\Delta t=0.005$ (110 steps per cycle) is temporally converged on this grid for all harmonics up to the third harmonic.

In summary, the nondimensional grid size necessary for the current numerical algorithm to accurately capture the physics of this flow is as follows: $\Delta z=0.1$ (32 points per transverse wave) and $\Delta x=0.01277$ (43 points per axial wave). The nondimensional time step necessary is $\Delta t=0.005$ (110 steps per cycle). This

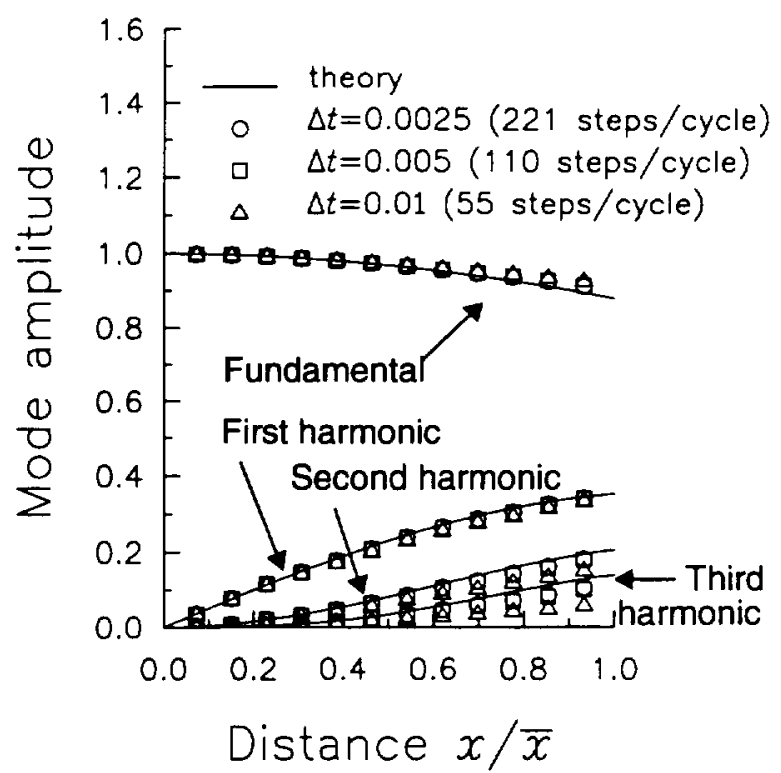

Fig. 7. Effect of time step on harmonics at $z=0$, with $\Delta x=0.01277$ and $\Delta z=0.1$.

baseline grid and time step are utilized to assess the effect of a sliding-zone interface.

Although not shown, a two-zone grid with zone 2 held stationary yields a solution that is identical to the singlezone solution. When zone 2 slides past zone 1 at a constant velocity, the solution becomes distorted as the zone speed increases, as shown in figure 8. In this plot, the theoretical predictions and the third harmonic are not shown, and the computed results are displayed as lines rather than symbols for clarity. The zonal interface is at $x / \bar{x}=0.5047$. Up to a speed of roughly $w_{\text {znne }}=6$ (nondimensionalized by the speed of sound), the solution is essentially unaffected. At $w_{\text {zone }} \geq 12$, however, the solution deteriorates significantly.

At a time step of $\Delta t=0.005$ and a zone speed of $w_{\text {zone }}=6$, zone 2 slides past one periodic variation in the acoustic data (i.e., the space over which the flow field is periodic in the $z$ direction) in approximately 107 time steps. This parameter can be used as a rule-ofthumb indicator of the limit on the time step when a sliding zone is present. Extensive investigation by the author (not all of which is reported here) indicates that if a zone is sliding past another zone and flow-field spatial variations in the direction of zone motion are present, then the time step must be set to allow approximately 80 or more time steps for the moving zone to traverse one period of that variation. This rule of thumb is summarized by the following equations:

$$
\left|\left(w_{z o n e}\right)_{\max }-w_{d a t a}\right| \approx \frac{z_{\text {period }}}{80 \Delta t}
$$

or

$$
\Delta t_{\max }=\frac{z_{\text {period }}}{80\left|w_{\text {zone }}-w_{\text {data }}\right|}
$$




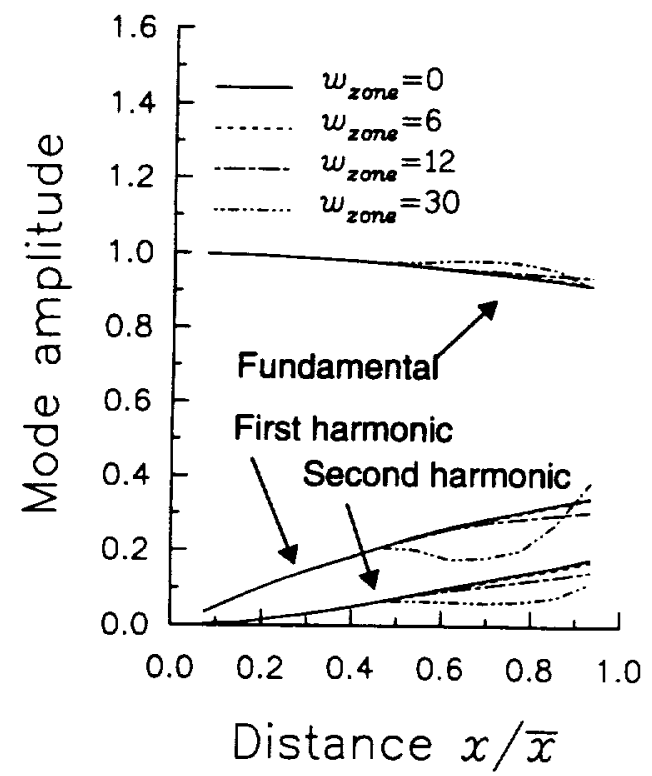

Fig. 8. Efrect of zone speed on harmonics at $z=0$, with $\Delta x=0.01277, \Delta z=0.1$, and $\Delta t=0.005$.

where $z_{\text {period }}$ is the spatial distance in the direction of zone motion over which the flow field varies by approximately one period and $w_{d a l}$ is the component of the acoustic-wave velocity parallel to the moving-zone interface. Because this rule of thumb is based only on qualitative estimates of acceptable error, it is best utilized as a guideline for establishing an initial estimate for the maximum time step or zone speed. Further refinement to the desired tolerance can be made through parametric studies.

In the case of axially-moving waves, $w_{\text {dara }}=0$. If the time step is fixed at $\Delta t=0.005$, then the maximum zone speed according to the guideline is roughly 8 . This conclusion is consistent with the results in figure 8 . If, on the other hand, the zone speed is fixed at $w_{\text {zone }}=30$, then the time step must be no larger than $\Delta t=0.0013$. The results obtained with $\Delta t=0.001$ and $w_{20}=30$ are contrasted with those obtained with $\Delta t=0.005$ and $w_{\text {zone }}=30$ in figures 9 and 10 . The former result shows little distortion of the pressure contours in zone 2 , whereas the latter shows large distortion. The oval contours should travel left to right only, with some compression, and then diminish past $x / \bar{x}=1$, where the grid is stretched.

This rule of thumb is independent of grid spacing in the direction of zone motion. Although not shown, twozone results with 17,33 , and 65 grid points in the $z$ direction yield nearly identical results when $\Delta t$ and $w$ are held fixed. Also, if zone 1 is clustered so that $\Delta z$ varies from 0.1 to 0.0001 while zone 2 retains uniform grid spacing, the results again follow the same rule of thumb. The number of $\tau$-TS multigrid subiterations currently employed (3) is sufficient to drive the $L_{2}$ norm of the equation for density down by at least 1.5 orders of magnitude for each time step. The use of six subiterations drives the residual down 3.5 orders for each time step, with no perceptible change in the solution.

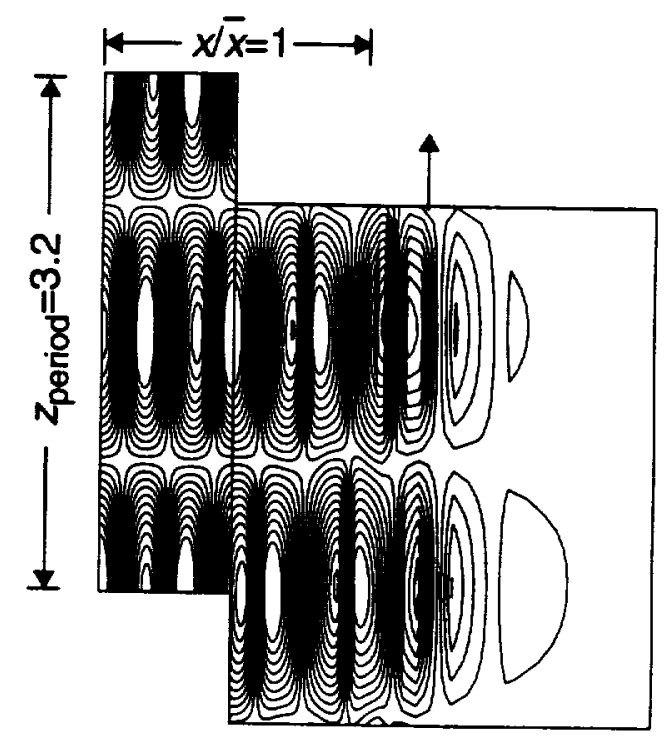

Fig. 9. Pressure contours with $\Delta t=0.001, T=3.6$, and $w_{\text {zane }}=30$.

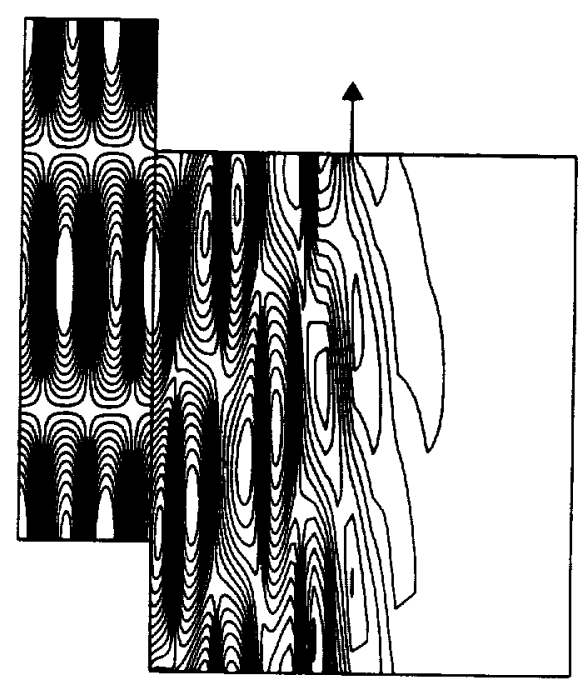

Fig. 10. Pressure contours with $\Delta t=0.005, T=3.6$, and $w_{\text {zone }}=30$.

\section{D Vibrating Plates}

The following 2D model problem is patterned after a computation by Huff. ${ }^{17}$ An infinite row of vibrating flat plates is simulated on the rectangular domain depicted in figure 11. The plates, of chord length $c^{\prime}$, are separat ed by height $h=h^{\prime} / c^{\prime}=1$. Slip-velocity (Euler) boundary conditions are applied on the plates, and periodic boundary conditions are applied elsewhere on the upper and lower boundaries. The left and right boundaries employ a characteristic boundary condition, and the grid is stretched in the $x$ direction past 
$x=x^{\prime} / c^{\prime}=5$. When used, a sliding-zone interface is employed at a location 0.5 chord length downstream of the trailing edge of the plates, which vibrate in phase with each other in accordance with

$$
z^{\prime}=h^{\prime} \sin \left(\omega^{\prime} t^{\prime}\right)
$$

where $\omega^{\prime}$ is the frequency in $\mathrm{rad} / \mathrm{s}$.

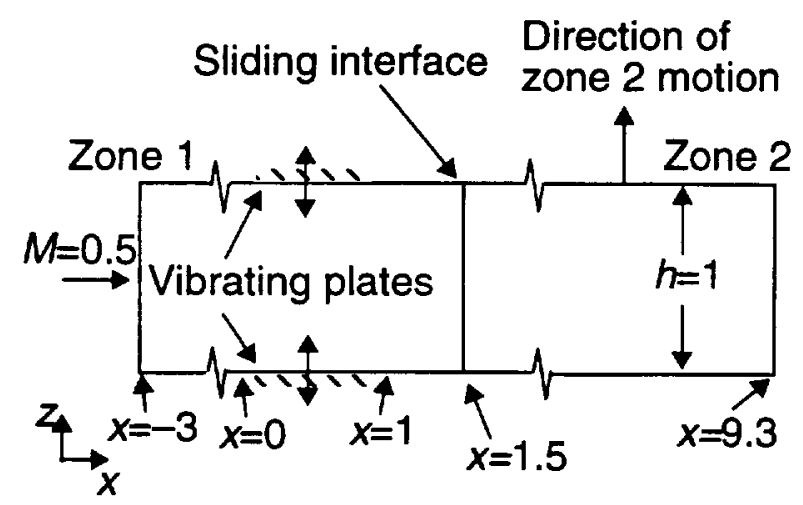

Fig. 11. Sketch of 2D vibrating-plate case.

If the lengths are nondimensionalized by $c^{\prime}$ and time by $c^{\prime} / a^{\prime}{ }_{\infty}$ and the reduced frequency is defined as

$$
k=\frac{\omega^{\prime}\left(c^{\prime} / 2\right)}{u_{\infty}^{\prime}}
$$

then the nondimensional equation that defines the plate vibration becomes

$$
z=h \sin \left(\frac{M_{\infty} k t}{2}\right)
$$

Here, $h$ is taken as $0.00004, k=8 \pi$, and $M_{\infty}=0.5$. Many acoustic waves generated by this test problem travel at an oblique angle in both directions relative to the sliding interface. At this frequency, approximately four complete cycles of pressure variation span the space between the plates. Hence, $z_{\text {period }} \approx 0.25$. A vertical uniform grid spacing of $\Delta z=0.0078125$ is used, which results in a spatial resolution of approximately 32 grid points per wave. The grid spacing in the axial direction is $\Delta x=0.03125$ between $x=-3$ and $x=5$ and is stretched past $x=5$. Although not shown, parametric studies indicate that this grid spacing is adequate to spatially resolve this flow field.

Because this model problem is based loosely on the types of acoustic waves that may be generated by vibrating stator vanes in an engine, computations are carried out with a fixed zone speed that is comparable to that of a typical maximum rotor tip speed. Hence, $w_{\text {zone }}=1.2$ is chosen; this value corresponds to a tip Mach number

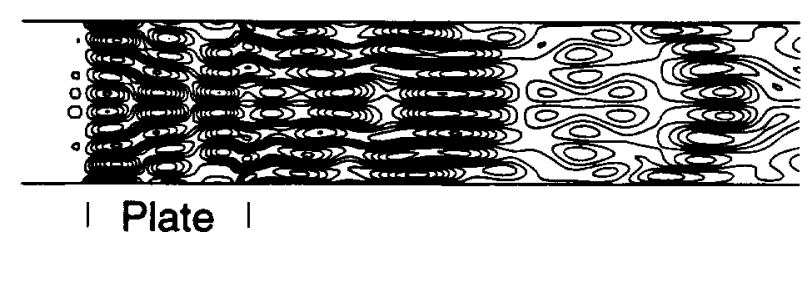

Fig. 12. Pressure contours with one zone, $\Delta t=0.00125$, and $T=5$.

of $M=1.2$. Equation (19), with $w_{\text {data }}= \pm 1$ (because the waves travel obliquely to the zone interface with a maximum speed equal to the speed of sound), is used to determine a maximum time step of $\Delta t_{\max }=0.0014$. Time-step studies (not shown) indicate that the time step necessary to adequately resolve the physics of this flow field is approximately $\Delta t=0.0025$, which corresponds to 400 time steps per period of plate oscillation. Hence, given the fixed zone speed of $w_{\text {zone }}=1.2$ in this case, the time-step constraint that is attributable to zone motion is more restrictive than the time-step constraint needed to adequately resolve the flow field.

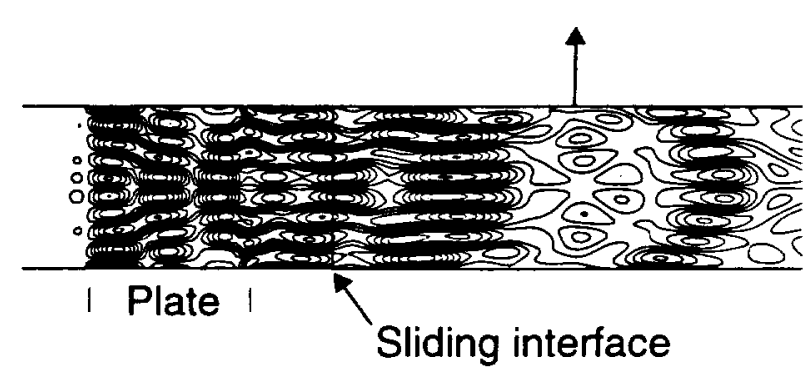

Fig. 13. Pressure contours with two zones, $\Delta t=0.00125$, $T=5$, and $w_{\text {:one }}=1.2$.

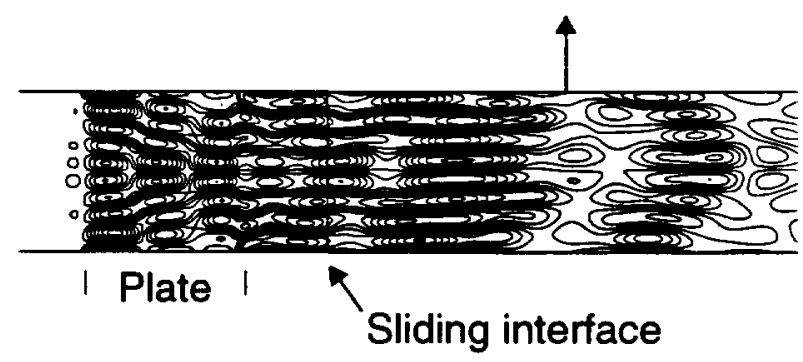

Fig. 14. Pressure contours with two zones, $\Delta t=0.0025$, $T=5$, and $w_{\text {zone }}=1.2$.

Because of the limitation caused by zone motion, a time step $\Delta t=0.00125$ is employed. Pressure contours (nondimensionalized by $\rho_{\infty}^{\prime} a_{-\infty}^{\prime 2}$ ) for a single zone are 
shown in figure 12. Contour levels range from 0.712 to 0.716 in steps of 0.0002 . Figures 13 and 14 show the results of using two zones, with time steps of $\Delta t=0.00125$ and $\Delta t=0.0025$, respectively. Results with the smaller time step are only slightly affected by the sliding-zone interface, whereas results with the larger time step show greater distortion of the acoustic waves after they have passed through the zone interface. Additional details of these differences are shown in figure 15 , which is a plot of pressure levels as a function of $x$ along a line at $z=0.75$.

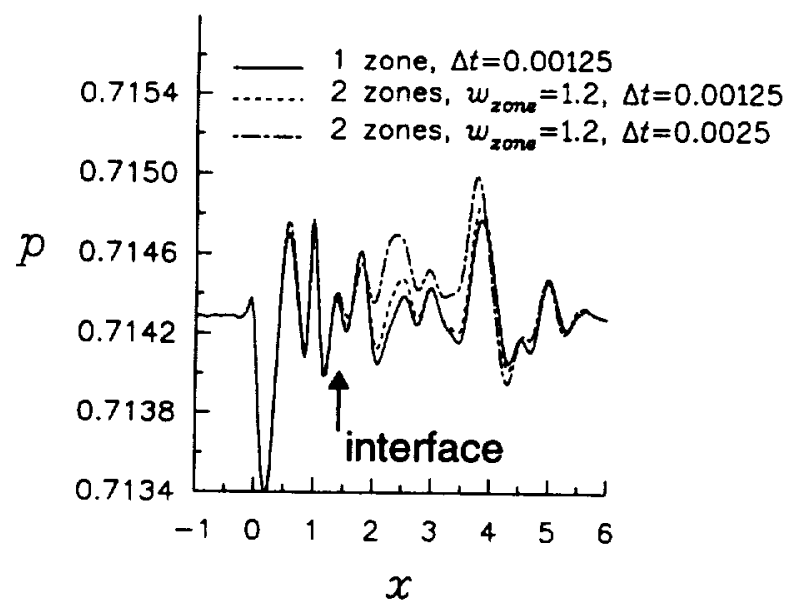

Fig. 15. Pressures along $z=0.75$ with $T=5$.

\section{D Rotor-Stator Interaction Modes}

To assess the passage of acoustic waves through a sliding-zone interface in a more realistic configuration, theoretical rotor-stator interaction modes are derived based on a 16-blade 20-vane NASA Langley model, ${ }^{18}$ with duct radius $r^{\prime}=0.1393 \mathrm{~m}$, Mach number $M=0.6$, and rotor speed $16,900 \mathrm{rpm}$. The theoretical levels of pressure perturbation are

$$
p^{\prime}\left(r^{\prime}, \theta, x^{\prime}\right)=A^{\prime} J_{m}\left(k_{r}^{\prime} r^{\prime}\right) \exp i\left[a^{\prime} k^{\prime} t^{\prime}-m \theta-k_{a}^{\prime} x^{\prime}\right]
$$

where $A^{\prime}$ is the magnitude of the perturbation, taken as $0.001 p_{0}{ }^{\prime}$ ( $p_{0}{ }^{\prime}$ is taken as $100,000 \mathrm{~Pa}$ ), and $J_{m}$ is the Bessel function of the $m$ th kind. One of the propagating modes (for a single blade passage frequency (BPF)) is the $(-4,1)$ mode, where $m=-4$ is the circumferential mode number and $n=1$ is the radial mode number. For a single $\mathrm{BPF}, k^{\prime}=87.97 \mathrm{~m}^{-1}$; for the $(-4,1)$ mode, $k_{r}^{\prime}=38.17 \mathrm{~m}^{-1}$ and $k_{a}^{\prime}=-211.37 \mathrm{~m}^{-1}$. This mode spins in the direction that is opposite to that of the rotor.

To test whether this acoustic mode can be propagated undistorted through a patched sliding-zone interface, a 3D time-accurate computation is performed in a cylindrical duct. Because the $(-4,1)$ mode is periodic over $\pi / 2$, the grid used is a quarter of a cylinder. The grid extends from $x^{\prime}=-0.5$ to $x^{\prime}=0 \mathrm{~m}$ and is divided into three zones. The middle zone, which extends from $x^{\prime}=-0.2786$ to $x^{\prime}=-0.1393 \mathrm{~m}$, can be rotated. Timedependent pressure perturbations are imposed at the downstream boundary (at $x^{\prime}=0$ ) with equation (23).
Based on grid studies (not shown), the grid that represents the best compromise between accuracy and efficiency for this case is $273 \times 33 \times 33$, with $\Delta x^{\prime}=0.00116$ $m$ in the unstretched portion of the mesh. This grid size yields 33 points per wave in the circumferential direction and approximately 25 points per wave in the axial direction, which is sufficient to propagate this mode through both downstream zones with reasonably minimal phase shift and attenuation. (Maximum pressure levels are computed to be approximately $\pm 35 \mathrm{~Pa}$, whereas the exact linear solution is $\pm 40 \mathrm{~Pa}$.) The radial and circumferential spacing is uniform, and the axial spacing is uniform in the two downstream zones (with 121 grid points each) and is stretched in the upstream zone. The grid, with every fourth grid point plotted in the axial and circumferential directions for clarity, is shown in figure 16. In this figure, the middle zone is shown in a rotated position.

Time-step studies (also not shown) indicate that at least 36-71 time steps per period are required to propagate this mode through both downstream zones with minimal attenuation and phase shift. Therefore, a nondimensional time step $\Delta t=0.001$ is employed, which corresponds to 71 time steps per period for BPF.

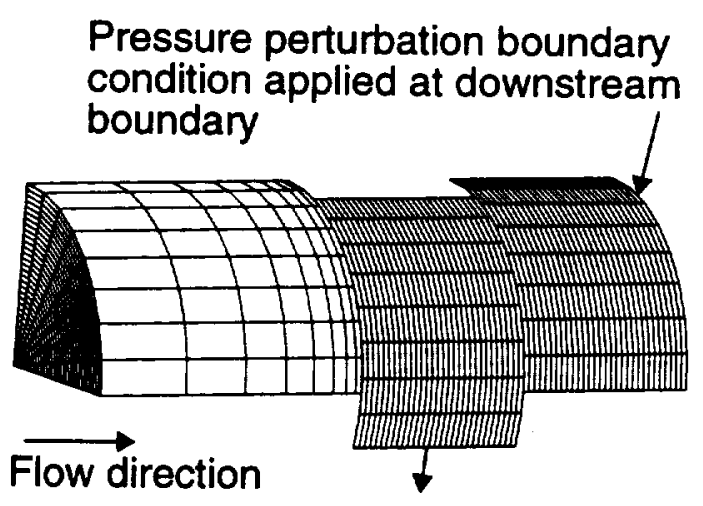

Fig. 16. $273 \times 33 \times 33$ duct grid (points removed for clarity).

The 3D duct acoustic modes show less sensitivity to a moving zone than the 2D test cases. A minimum of approximately 40 (rather than 80 ) time steps is required for the moving zone to pass one spatially periodic variation in the flow field. Therefore, for rotating zones, equations (18) and (19) are modified to read as

$$
\left|\left(\dot{\theta}_{z o n e}\right)_{\max }-\dot{\theta}_{\text {daral }}\right|=\frac{\theta_{\text {period }}}{40 \Delta t}
$$

or

$$
\Delta t_{\max }=\frac{\theta_{\text {period }}}{40\left|\dot{\theta}_{\text {zone }}-\dot{\theta}_{\text {data }}\right|}
$$

A fixed moving-zone speed of $\dot{\theta}_{\text {zone }}=16,900 \mathrm{rpm}$ results in a maximum allowable nondimensional time step of $\Delta l_{\max }=0.0014 \quad$ (with $\theta_{\text {period }}=90^{\circ}$ and 
$\left.\dot{\theta}_{\text {dasa }}=-67,600 \mathrm{rpm}\right)$. This time-step limitation that results from zone motion is slightly higher than the time step of $\Delta t=0.001$ necessary to resolve the physics of the flow (regardless of zone motion). Therefore, for this case, the constraint due to the numerical resolution of the physics is more restrictive than the constraint due to zone motion, and the zone motion is expected to have little or no discernible effect on the temporally resolved solution.

The acoustic waves generated by the downstream perturbation propagate upstream in a spiral manner (i.e., like a "barber pole"). A total nondimensional time of $T=0.8$ is sufficient to achieve a periodic solution through the two downstream zones. Figure 17 shows pressure perturbation levels as a function of $x^{\prime}$ along the duct wall at a $\theta=$ Constant location for a nonrotating zone and for a zone that is rotating at $16,900 \mathrm{rpm}$. Results are essentially identical for both cases, which indicates, as expected, that the sliding-zone interface does not distort the $(-4,1)$ mode acoustic waves with this grid and time step. Each computation requires approximately $4 \mathrm{hr}$ of CPU time on NASA Langley Research Center's CRAY Y-MP computer.

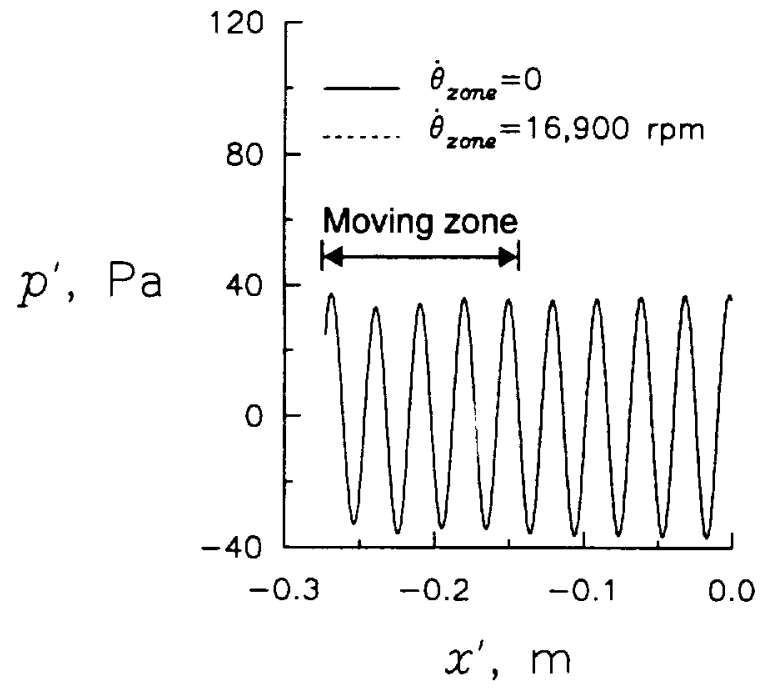

Fig. 17. Pressures along duct wall with $\Delta t=0.001$ and $T=0.8$.

Figure 18 shows the effect of increasing the zone rotation speed. Tripling the rotation speed results in negligible distortion, whereas quintupling the speed changes the character of the solution upstream of the movingzone interface. With equation (24), a time step of $\Delta t=0.001$ allows for a maximum zone speed of 53,113 rpm. This maximum speed is consistent with the results in the figure. Five subiterations are required for the higher zone speeds.

Other propagating modes are investigated by constructing a $241 \times 65 \times 65$ grid similar to the one depicted in figure 16 , except that $\Delta x^{\prime}=0.000481 \mathrm{~m}$ and the two downstream zones each extend only $x^{\prime}=0.05 \mathrm{~m}$. This shorter axial extent allows all modes of interest to propagate through both downstream zones in a reasonable computational time. This grid spacing yields between

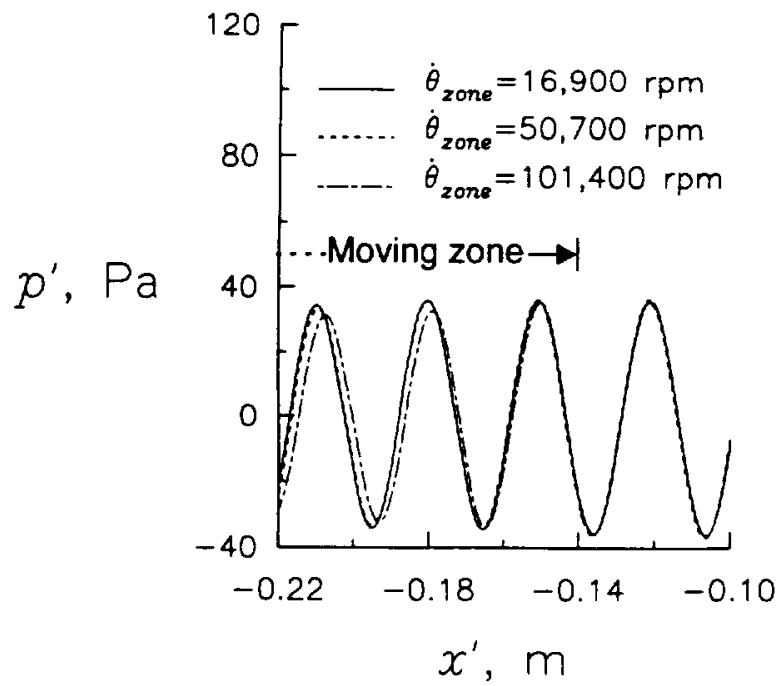

Fig. 18. Effect of zone speed on pressures along duct wall with $\Delta t=0.001$ and $T=0.8$.

10-65 points per wave in the circumferential direction and 31-82 points per wave in the axial direction, depending on the mode. The computation is performed by combining 10 different propagating modes at the downstream boundary, with varying acoustical strengths. These modes are described in Table 1. Included in the table is the acoustical strength $A^{\prime}$ that is used in the computation; also included is the theoretical cutoff ratio of each mode. Note that these 10 modes are a representative sample of the BPF and 2 BPF propagating modes in the NASA Langley Research Center model. ${ }^{18}$

Table 1 Computed Modes in 10-Mode Combined Computation

\begin{tabular}{cccccc}
\hline \hline Mode & $k^{\prime}, \mathrm{m}^{-1}$ & $k_{r}{ }^{\prime}, \mathrm{m}^{-1}$ & $k_{a}{ }^{\prime}, \mathrm{m}^{-1}$ & $A^{\prime}, \mathrm{Pa}$ & cutoff \\
\hline$(-4,1)$ & 87.97 & 38.17 & -211.37 & 30 & 2.88 \\
$(-4,2)$ & 87.97 & 66.64 & -191.80 & 60 & 1.65 \\
$(-4,3)$ & 87.97 & 91.04 & -159.55 & 100 & 1.21 \\
$(-8,1)$ & 175.93 & 69.26 & -425.84 & 30 & 3.18 \\
$(-8,3)$ & 175.93 & 127.60 & -388.83 & 60 & 1.72 \\
$(-8,6)$ & 175.93 & 200.21 & -278.67 & 100 & 1.10 \\
$(12,1)$ & 175.93 & 99.63 & -410.00 & 30 & 2.21 \\
$(12,4)$ & 175.93 & 188.41 & -306.70 & 60 & 1.17 \\
$(12,5)$ & 175.93 & 213.42 & -231.27 & 100 & 1.03 \\
$(-28,1)$ & 175.93 & 218.80 & -192.69 & 150 & 1.01 \\
\hline \hline
\end{tabular}

Computations are performed both with and without middle zone rotation. For the $m=-4,-8,12$, and -28 modes, $\theta_{\text {period }}$ is equal to $90^{\circ}, 45^{\circ}, 30^{\circ}$, and $12.85714^{\circ}$, respectively, and $\dot{\theta}_{\text {dara }}$ is equal to $-67,600 \mathrm{rpm},-67,600$ $\mathrm{rpm},+45,066 \mathrm{rpm}$, and $-19,314 \mathrm{rpm}$, respectively. With equation (25), the time step recommended for a minimal effect of zone motion at $\dot{\theta}_{\text {zone }}=16,900 \mathrm{rpm}$ is $\Delta t_{\text {max }}=0.0014,0.0007,0.0014$, and 0.0005 , respectively. However, in the interest of reducing the computational time, a nondimensional time step of $\Delta t=0.001$ is employed, which yields 71 time steps per period for sin- 
gle BPF and 36 for 2 BPF. Because the $\Delta t_{\max }$ from equation (25) is exceeded for several modes, some effects attributable to the moving zone can be expected. Computations are run to a nondimensional time of $T=0.75$. The computations require $13 \mathrm{hr}$ of CPU time on NASA Langley Research Center's CRAY Y-MP computer or $5.8 \mathrm{hr}$ on the NAS CRAY C-90 computer. Approximately 50 million words of memory are required. Results are analyzed by decomposing the computed solutions at various axial stations into individual modal amplitudes and comparing these amplitudes with the theoretical (undiminished) pressure amplitude.

Results for the $m=-4,-8,12$, and -28 modes are shown in figures 19-22. Note that the numerical algorithm, with this grid and time step, has difficulty propagating those modes that have the lowest cutoff ratios, regardless of whether a moving zone is employed. In general, the lower the cutoff ratio, the more the acoustic wave is attenuated. Further studies need to be performed to determine the grid and time-step requirements for all modes of interest. Overall, the effects of the slidingzone interface on the transmission of acoustic modes are very slight; the least effect occurs for the $m=-4$ modes.

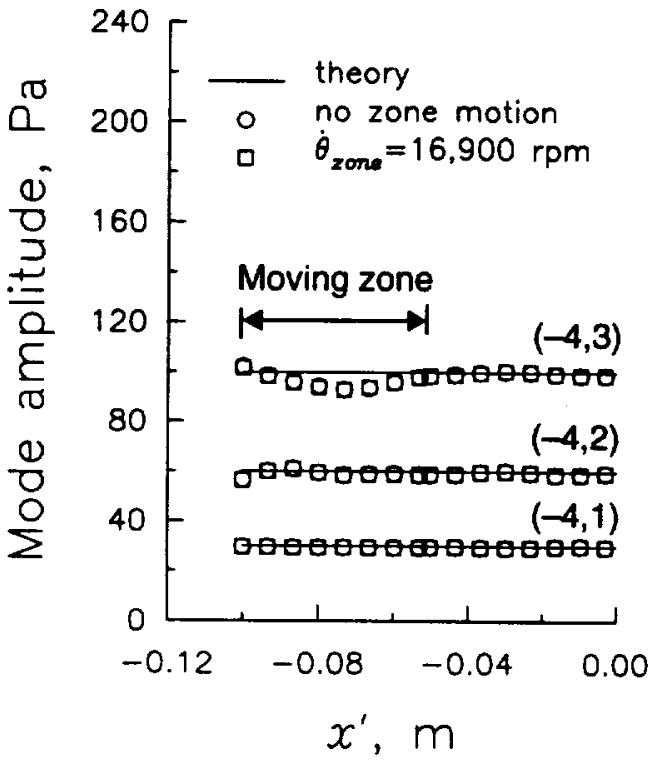

Fig. 19. Acoustic amplitudes for $m=-4$ modes in 10-mode combined computation.

\section{Conclusions}

Patched sliding-zone interfaces, in combination with the time-accurate Euler and Navier-Stokes equations, are employed for aerodynamic computations of rotorstator interactions (e.g., refs. 4-7). However, before codes with sliding interfaces can be used as a major part of any noise-prediction effort, the accuracy with which acoustic waves are passed through the sliding-zone interfaces must be assessed. Preliminary findings indicate that an important factor that contributes to the accuracy of acoustic-wave passage is the number of time steps required for the sliding zone to move past spatial varia-

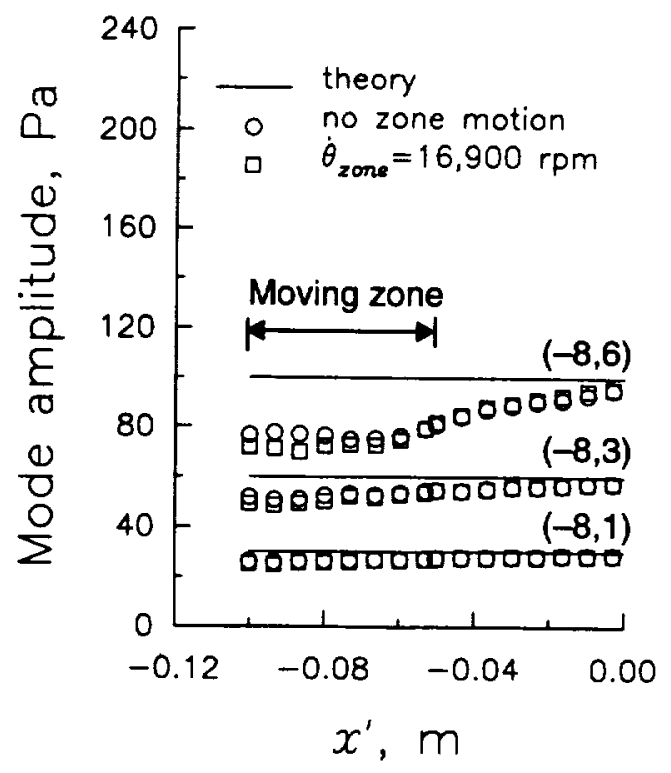

Fig. 20. Acoustic amplitudes for $m=-8$ modes in 10-mode combined computation.

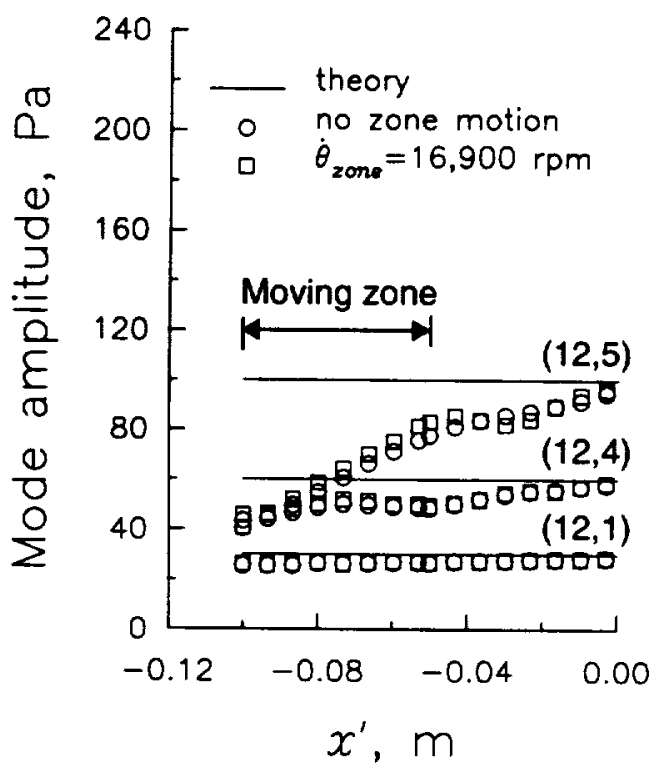

Fig. 21. Acoustic amplitudes for $m=12$ modes in 10-mode combined computation.

tions in the flow field that exist in the direction parallel to the sliding-zone interface (e.g., circumferential variations for duct flows). To avoid unreasonable distortions, at least 40-80 time steps are required for the sliding zone to pass one period of the spatial variation. If the time step is too large, then distortions in the acoustic waves result. This rule of thumb has been demonstrated for two simple two-dimensional test problems, as well as for a three-dimensional-duct test problem. 


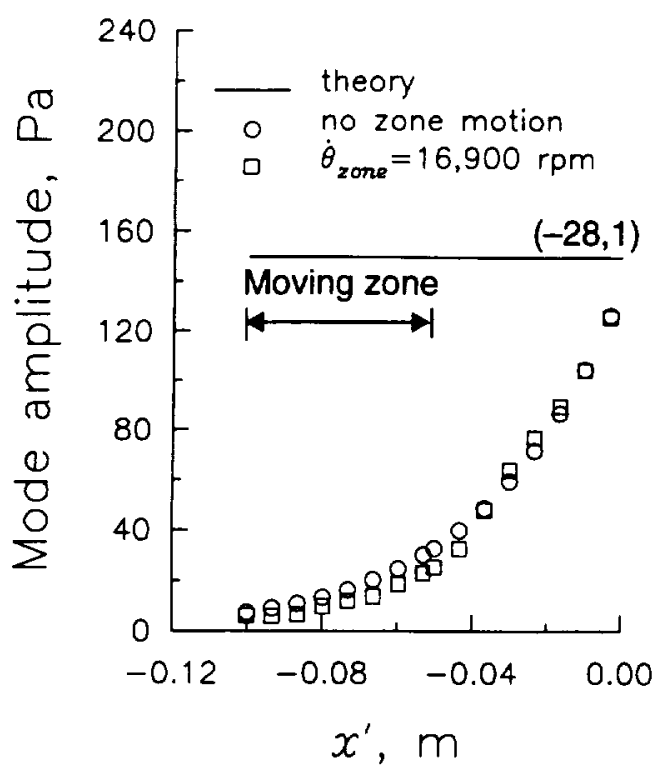

Fig. 22. Acoustic amplitudes for $m=-28$ mode in 10-mode combined computation.

\section{Acknowledgments}

The author acknowledges R. Biedron, of AS\&M, Inc., Hampton, VA, for coding the dynamic patched interface routines and for general CFL 3D code support, as well as for helpful discussions throughout the investigation. Also, the author thanks the other members of the Langley Ducted Fan Noise Prediction Team for their assistance: leader F. Farassat, C. Hsu, P. Spence, and M. Dunn. Thanks are also due to $M$. Janus at Mississippi State University for many helpful discussions and to D. Huff at NASA Lewis Research Center for sparking the initial interest in this study.

\section{Beferences}

${ }^{1}$ Jameson, A., "Time Dependent Calculations Using Multigrid, with Applications to Unsteady Flows Past Airfoils and Wings," AIAA 91-1596, 1991.

${ }^{2}$ Melson, D., Sanetrik, M., and Atkins, H., "Time-Accurate Navier-Stokes Calculations with Multigrid Acceleration," Sixth Copper Mountain Conference on Multigrid Methods, Part 2, NASA CP-3224, 1993, pp. 423-437. Also in Llth AIAA CFD Conference, Part 2, Orlando, Florida, July 1993, pp. 1041-1042.

${ }^{3}$ Rumsey, C., Sanetrik, M., Biedron, R., Melson, N., and Parlette, E., "Efficiency and Accuracy of Time-Accurate Turbulent Navier-Stokes Computations," AIAA 95-1835-CP, 13th AIAA Applied Aerodynamics Conference, 1995.

${ }^{4}$ Rai, M., "Three-Dimensional Navier-Stokes Simulations of Turbine Rotor-Stator Interaction, Parts I and II" Journal of Propulsion and Power, Vol. 5, No. 3, 1989 , pp. 307-319.

${ }^{5}$ Gundy-Burlet, K., Rai, M., and Dring, R., "Two-Dimensional Computations of Multi-Stage Compressor Flows Using a Zonal Approach," AIAA 89-2452, 1989.
${ }^{6} \mathrm{Hall}, \mathrm{E}$. and Delaney, R., "Investigation of Advanced Counterrotation Blade Configuration Concepts for High Speed Turboprop Systems," NASA CR 187126, 1993.

${ }^{7}$ Chen, C. and Chakravarthy, S., "Calculation of Unsteady Rotor/Stator Interaction,” AIAA 90-1544, 1990.

${ }^{8}$ Janus, M. and Whitfield, D., "Counterrotating PropFan Simulations which Feature a Relative-Motion Multiblock Grid Decomposition Enabling Arbitrary Time Steps," AIAA 90-0687, 1990.

'Rangwalla, A. and Rai, M., "A Numerical Analysis of Tonal Acoustics in Rotor-Stator Interactions," Journal of Fluids and Structures, Vol. 7, 1993, pp. 611-637.

"Thomas, J., Krist, S., and Anderson, W., "NavierStokes Computations of Vortical Flows Over High Aspect-Ratio Wings," AIAA Journal, Vol. 28, No. 2, 1990, pp. 205-212.

${ }^{1}$ Roe, P., "Approximate Riemann Solvers, Parameter Vectors, and Difference Schemes," Journal of Computational Physics, Vol. 43, 1981, pp. 357-372.

12Van Leer, B., "Flux Vector Splitting for the Euler Equations," Lecture Notes in Physics, Vol. 170, 1982, pp. 501-512.

13Venkateswaran, S. and Merkle, C., "Dual Time Stepping and Preconditioning for Unsteady Computations," AIAA 95-0078, 1995.

${ }^{14}$ Biedron, R. and Thomas, J., "A Generalized Patched-Grid Algorithm with Application to the F-18 Forebody with Actuated Control Strake," Computing Systems in Engineering, Computational Technology for Flight Vehicles, Vol. 1, Nos. 2-4, 1990, pp. 563-576.

${ }^{15}$ Khan, M., "Computations of Aeroacoustic Fields Using Finite Volume Method," Computational Acoustics: Algorithms and Methods, (D. Lee, R.L. Sternberg, M.H. Schultz, eds.), Elsevier Science Publishers B.V., North Holland, 1988, pp. 83-101.

${ }^{16}$ Pierce, A., Acoustics, An Introduction to its Physical Principles and Applications, Acoustical Society of America, American Institute of Physics, New York, 1989, pp. 571-573.

${ }^{17}$ Huff, D., "Pressure Wave Propagation Studies for Oscillating Cascades," AIAA 92-0145, 1992. Also NASA TM-105406, 1992.

18Thomas, R., Gerhold, C., Farassat, F., Santa Maria, O., Nuckolls, W., and DeVilbiss, D., "Far Field Noise of the 12 Inch Advanced Ducted Propeller Simulator," AIAA 95-0722, 1995. 


